\title{
Space-Time Anomaly Characteristics of Strain Field before the Ms $\geq 7.0$ Earthquakes, In Mainland China
}

Guofu Luo ( $\nabla$ luoguofu_05@163.com )

China Seismological Bureau: China Earthquake Administration

\section{Ding Fenghe}

National Earthquake Bureau: China Earthquake Administration

\section{Yang Mingzhi}

National Earthquake Bureau: China Earthquake Administration

\section{Research Letter}

Keywords: seismic strain field, China mainland, natural orthogonal function expansion method, spacetime anomaly

Posted Date: May 6th, 2021

DOI: https://doi.org/10.21203/rs.3.rs-458696/v1

License: (c) (i) This work is licensed under a Creative Commons Attribution 4.0 International License. Read Full License 


\title{
Space-Time Anomaly Characteristics of Strain Field before the $M s \geqslant 7.0$ Earthquakes, In Mainland China
}

\author{
Luo Guofu*, Ding Fenghe, Yang Mingzhi \\ Seismological Bureau of Ningxia Hui Autonomous Region, Ningxia, Yinchuan 750001 \\ * Corresponding author. Email: luoguofu_05@163.com
}

\begin{abstract}
Acknowledgments: We would like to thank research fellow Song Zhiping at the seismic network center of China Seismological Bureau, and research fellow Yang Liming at the seismological Bureau of Qinghai Province for their guidance and assistance in writing this paper.

Author Contributions: All authors contributed to the conception and design of this study. Luo Guofu, Ding Fenghe, and Yang Mingzhi prepared the material, collected the data, and performed the analysis . The first draft of the manuscript was written by Luo Guofu, and all authors provided their feedback on previous versions of the manuscript. All authors have read and approved the final manuscript.
\end{abstract}

Abstract: Seismologists focus on analyzing and capturing medium and short-term impending anomalies of medium and small earthquakes before large earthquakes. The natural orthogonal expansion method can effectively extract small and medium seismicity anomalies in the epicentral region before large earthquakes, which can improve the prediction of large earthquakes. In this study, the strain range of small earthquakes that occurred in the Chinese mainland before 1980 , specifically ten $M \mathrm{~s} \geq 7.0$ earthquakes, were calculated, and the temporal and spatial anomalies of the strain fields before the large earthquakes were extracted. The active faults in each large earthquake area were analyzed to identify the dangerous areas (Epicenter of earthquake) of the large earthquake. In addition, compared with the seismic energy field method, the natural orthogonal expansion method effectively captured the short-term and impending anomalies in the seismic strain field before large earthquakes. The results of this study can have a significant impact on the analysis of anomalies by strain field, and make an outstanding contribution to the study of large earthquake prediction.

Keywords: seismic strain field; China mainland; natural orthogonal function expansion method; space-time anomaly

\section{Introduction}

The natural orthogonal function expansion method was first applied in meteorological research(Cyrillic 1971, Ma et al. 1993). It mainly based on the accuracy of temperature and barometric data requirements observed by meteorological stations. The convergence of this method is applied to the meteorological typical field, and its future weather conditions are analyzed and predicted. The advantage of the natural orthogonal function expansion method is that the function cluster can be determined from the correlation of the meteorological typical field itself and some natural conditions without giving a function cluster in advance. Such a function is called a natural orthogonal function. Similar to meteorological prediction, earthquake prediction research is also applicable to this method. It is well known that seismic activity is also a random natural phenomenon. The occurrence and occurrence of earthquakes is the process of strain accumulation and release around the source. Using seismic strain as an independent variable, applying natural orthogonal function The method is to reveal the relationship between the earthquake anomaly caused by the space-time evolution of seismic strain and the large earthquake, so as to seek to explore the earthquake prediction. Therefore, this paper attempts to apply the natural orthogonal expansion method to study the space-time anomaly characteristics of the strain field before the $M s \geq 7$ earthquake in China. This solves the problem of large 
earthquake prediction.

Seismic energy fields, in particular, have been studied intensively in recent years, this is the first time in China, but there are no papers on the application of relevant methods in the field of seismic strain field research in the United States, Europe and Japan. For example, the random field theory was used for the first time by Yang et al. (2004) to study how temporal anomalies in seismic energy fields are related to strong earthquakes in Ningxia. Luo et al. (2005) conducted a systematic analysis on the relationship between spatial and temporal seismic energy field anomalies and moderate-to-strong earthquakes in Yunnan, based on the seismic energy field of Yunnan since 1975. Luo (2011) and Yang (2011, 2012) discovered medium-term and short-term temporal and spatial anomalies in the seismic energy field of Wenchuan prior to the 2008 Ms 8.0 Wenchuan Earthquake, which corresponded closely to the earthquake event. Ma et al. (2012) analyzed the pre-quake temporal changes in the seismic energy field of the area around the epicenter of the 2010 Ms 7.1 Yushu Earthquake and the Yushu Fault, and they found that spatiotemporal energy field anomalies preceded the earthquake by approximately half a year.Yang et al. (2013) reviewed the pre-quake temporal factors of seismic activity fields around the epicenters of $30 \mathrm{Ms} \geq 6.0$ earthquakes in China and found that most of these earthquakes were preceded by significant temporal anomalies. Yang et al. (2017) studied the strain fields of $M \mathrm{~s} \geq 6.0$ earthquakes in China and found that the temporal anomalies of seismic strain fields are more useful as earthquake predictors than those of the seismic energy fields. Luo et al. (2018) studied the pre-quake seismic strain field of the 2017 Ms 7.0 Jiuzhaigou Earthquake and compared the temporal evolution of the strain anomalies of this earthquake with that of the 1976 Ms 7.2 Songpan - Pingwu Earthquake .Luo et al. (2019) studied how the seismic strain field of the $M \mathrm{~s} 8.0$ Wenchuan Earthquake affected the subsequent $M \mathrm{~s} \geq 6.0$ earthquakes in the Longmenshan Fault and other nearby faults, by investigating the interactions between strong earthquakes from the perspective of seismic strain evolution.

In this work, the natural orthogonal function expansion method is used to calculate the seismic activity strain field of each study area before the earthquake in Chinese mainland, and the time factor anomaly of the main strain field and the relationship between time anomaly and strong earthquake will be analyzed. Spatial anomaly, the change of grid space value in the study area, the main reason for causing time anomaly is the occurrence of significant foreshock or seismic activity anomaly in the study unit cell grid. How to capture the foreshock activity may be one of the path to break through the earthquake prediction which is the innovation point of this paper, and it could provide a practical reference for future earthquake prediction researchers.

\section{Method and principles}

Temperature and pressure variables in meteorological observations are the functions of space-time position, similar to the time and spatial functions of earthquakes. These functions comprise the earthquake strain field, $\boldsymbol{S}$. A random field may then be created by gridding the study area according to its local Seismic activity level, that is, by dividing the study area, $\Delta S$, into $\mathrm{n}$ elements of equal areas, such that $\Delta S=\Delta x \times \Delta y$. The central coordinates of each element are $\left(x_{j}, y_{j}\right)(j=1,2, \ldots, n)$. The observation time, $t_{i}$, is then divided into $\mathrm{m}$ intervals of length $\Delta t$, such that $t_{i}=\Delta t \times i(i=1,2, \ldots, m)$. The observed seismic activity strain of each element in each time interval is then calculated and recorded as $S_{i j}$; these values are the function field of the spatiotemporal coordinates $\left(x_{i}, y_{i}, t_{j}\right)(i, j=1$, $2, \ldots, n)$.

In this work, $\boldsymbol{E}$ is the energy of the seismic activity applied in the gridding of the study area, and $\sqrt{E}$ is the square root of the energy. ie $\sqrt{E}=c \varepsilon$ ( $c$ is the source-related parameter of the earthquake in 
the study area, and the cumulative strain parameter of the earthquake in the source area). These two parameters reflect the variation of the strain field in the source area to some extent. It is expressed as $S=\sum_{i} \sqrt{E_{i}}$, and has the following matrix representation

$$
S=\left[\begin{array}{cccc}
S_{11} & S_{12} & \mathrm{~L} & S_{1 n} \\
S_{21} & S_{22} & \mathrm{~L} & S_{2 n} \\
\mathrm{M} & \mathrm{M} & \mathrm{M} & \mathrm{M} \\
S_{m 1} & S_{m 2} & \mathrm{~L} & S_{m n}
\end{array}\right]
$$

In this equation, $S_{i j}(i=1,2, \ldots, m, j=1,2, \ldots, n)$ is the cumulative earthquake strain of the $j$-th grid (spatial factor) during the $i$-th time interval (temporal factor). A natural orthogonal expansion is then performed on $S$ by decomposing the matrix into the sum of multiplications between orthogonal spatial functions $x$ and orthogonal temporal functions $T$, that is,

$$
S_{i j}=\sum_{p=1}^{n} T_{i p} X_{p j}\left\{\begin{array}{cc}
i=1,2, \mathrm{~L} & , m \\
j=1,2, \mathrm{~L} & , n
\end{array} .\right.
$$

These functions are orthogonal and normalized, and they satisfy the conditions

$$
\begin{aligned}
& \sum_{j=1}^{n} x_{k j} x_{l j}= \begin{cases}0 & k \neq l \\
1 & k=l\end{cases} \\
& \sum_{i=1}^{m} T_{i k} T_{i l}= \begin{cases}0 & k \neq l \\
\lambda_{k} & k=l\end{cases}
\end{aligned}
$$

Let there be a matrix $R$ such that $R=S^{\prime} S$. By solving for the characteristic equation of $R$, that is,

$$
\left[\begin{array}{cccc}
R_{11} & R_{12} & \Lambda & R_{1 n} \\
R_{21} & R_{22} & \Lambda & R_{2 n} \\
\Lambda & \Lambda & \Lambda & \Lambda \\
R_{n 1} & R_{n 2} & \Lambda & R_{n n}
\end{array}\right]\left[\begin{array}{c}
x_{1} \\
x_{2} \\
\Lambda \\
x_{n}
\end{array}\right]=\lambda\left[\begin{array}{c}
x_{1} \\
x_{2} \\
\Lambda \\
x_{n}
\end{array}\right]
$$

we obtain the eigenvectors $\ddot{x}_{k}$ and eigenvalues $\lambda_{k}(k=1,2, \ldots, n)$. The temporal factors may then be expressed as

$$
\stackrel{\mathrm{v}}{T_{k}}=S \mathrm{v}_{k} \quad k=1,2, \Lambda n
$$

The eigenvectors $\ddot{x}_{k}$ (strain field) represent the spatial distribution of the earthquake strains in each field, whereas the temporal factor $\tilde{T}_{k}$ represents the time dependence of the strain field $\varkappa_{k}$ and reflects the dynamics of each time strain field. The eigenvectors corresponding to the first few eigenvalues of the matrix $\boldsymbol{R}$ (which are arranged in decreasing order) are sufficiently precise to fit the entire strain field. In other words, we can approximate the entire strain field by superimposing the first few eigenvectors (strain fields), and the changes that occur in the first few strain fields represent the spatiotemporal characteristics of the strain field corresponding to the study area. If the sum of all $n$ eigenvalues is $b_{0}$, the precision of fit between the first $l$ strain fields and the entire field is denoted as $r_{l}$, which is defined as 


$$
r_{l}=\sum_{p=1}^{l} \lambda_{p} / b_{0} .
$$

In this equation, $\lambda_{p}$ is the $p$-th characteristic root.

In summary, natural orthogonal expansion condenses most of the information carried by an earthquake strain field into the "primary" strain fields that correspond to its largest eigenvalues. Strain fields that are either invariant or poorly correlated with large quakes may then be excluded, thus simplifying the problem to the anomalies of the first few strain fields.

\section{Data and case examples}

\subsection{Earthquake data}

The earthquake data used in this study were obtained from the official national earthquake catalog provided by the China Earthquake Networks Center. The $b$-values of the study areas that correspond to the epicenters of ten $M_{\mathrm{s}} \geq 7.0$ earthquakes were estimated from the earthquake catalog and their minimum magnitude of completeness, $M c$. To ensure that the selected dataset will adequately reflect the background seismicity of a region as well as the seismogenic processes of large earthquakes, the earthquakes between $M c$ and $M_{\mathrm{L}} 5.4\left(M_{\mathrm{s}} 5.0\right)$ were included in the dataset. In principle, the aftershocks of each study area will also be included in the dataset. However, if the study areas of the $M_{\mathrm{s}} \geq 7.0$ earthquakes overlap with each other, it is necessary to eliminate the aftershocks of the earlier $M_{\mathrm{s}} \geq 7.0$ earthquake to avoid affecting the later $M_{\mathrm{s}} \geq 7.0$ earthquake. For example, the study areas of the $2008 M_{\mathrm{s}}$ 8.0 Wenchuan Earthquake and $2013 M_{\mathrm{s}} 7.0$ Lushan Earthquake overlap with each other, in this case, it was necessary to omit the aftershocks of the $M_{\mathrm{s}} 8.0$ Wenchuan Earthquake from the dataset. The grids should be configured in a manner that will adequately reflect the characteristics of the earthquake frequency distribution of each study area. Based on a multitude of trials, it was determined that the study areas (Table 1) should be discretized using $0.5^{\circ} \times 0.5^{\circ}$ square grid elements and a time interval of three months. Earthquake strain matrices, $S$, were constructed for each study area using Eq. (1). These matrices were then analyzed by natural orthogonal expansion. The $\boldsymbol{R}$ matrices were then solved to obtain the eigenvalues of the strain field, primary fields that correspond to these eigenvalues, temporal factors of these primary fields, and strains of the spatial grids of each study area.

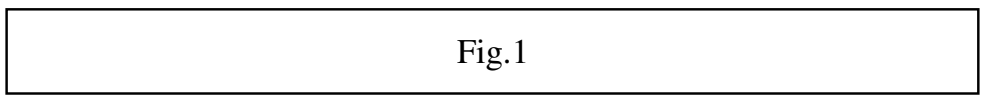

Fig. 1 Study areas of ten $M_{\mathrm{s}} \geq 7.0$ earthquakes in mainland China

In this work, the natural orthogonal function is used to analyze the regional strain field of all $M_{\mathrm{s}}$ $\geq 7.0$ earthquakes in mainland China since 1980 (Fig. 1). However, due to disparities in the precision and sensitivity of the national seismograph network (especially in Tibet and parts of Qinghai, where the network is poorly developed), the seismic observation data of the following earthquakes were not sufficiently complete for the purposes of this study: $M_{\mathrm{s}} 7.5$ Earthquake in Mani, Tibet, on 8 November 1997, and $M_{\mathrm{s}} 8.1$ earthquake to the west of the Kunlun Pass on 14 November 2001. Therefore, the frequency fields of these earthquakes were not computed in this work.

\subsection{Seismic parameters and computational accuracy}

Considering the limited length of the article, this work takes the calculation parameters of the Xinjiang Wuqia $M_{\mathrm{s}} 7.1$ earthquake on August 23, 1985 as an example, and gives the calculation process The results of other 9 calculations of earthquakes with magnitude 7 or above in China are shown in Table 1. The study area of Wuqia $M_{\mathrm{s}} 7.1$ earthquake is $\left(74.5^{\circ}-77.5^{\circ} \mathrm{E}, 39^{\circ}-41.5^{\circ} \mathrm{N}\right)$, and the time period 
for selecting seismic data is from January 1, 1975 to August 22, 1985. The data was tested for b-value, The $M c$ of this area was determined to be $M_{\mathrm{L}}=2.7$. The study area was divided into $0.5^{\circ} \times 0.5^{\circ}$ grids, with a time interval of $\Delta t=1 / 4$ year ( 3 months), and the sliding step is $d t=1 / 12$ year ( 1 month). The earthquake strain field of the $n$ grids $(n=30)$ and $m$ time intervals $(m=125)$ was then computed. When $l$ $=4$, the first four frequency fields encompass $83.29 \%$ of the entire strain field, which is equivalent to all the pre-quake strain field anomalies of the $M_{\mathrm{s}} 7.1$ Wuqia Earthquake being condensed within these four primary strain fields. In other words, the anomalies in the first four strain fields were equivalent to all the anomalies of the study area. The parameters of the other nine $M_{\mathrm{s}} \geq 7.0$ earthquakes and their computed results are shown in Table 1.

Table 1 Parameters and computed frequency fields of nine $M s \geq 7.0$ earthquakes in mainland China

Table 1

The strain field parameters computed for ten $M s \geq 7.0$ earthquakes that occurred in mainland China since 1980 are shown in Table 1, This table includes the selected earthquakes and their spatial and temporal ranges, the magnitudes of the weak earthquakes included in the strain field, the number of computational grids and time intervals, the magnitudes of the first four eigenvalues, and the accuracy of fit between the first four strain fields and the entire field.

Fig.2

Fig.2 Temporal factors of the first four strain fields of ten $M_{\mathrm{s}} \geq 7.0$ earthquakes

The variation of the strain field with time before the large earthquake is shown in Fig.2. By studying the characteristics of the seismic strain field of ten earthquakes of the $M s \geq 7.0$, the time range of the strain field of each seismic region is analyzed. The magnitude and variance of the anomaly change before ten earthquakes are identified, and the mid-term anomaly, short-term anomaly and impending earthquake anomaly are identified. Further clarify the main strain field causing the abnormal change of the time factor (Table 2), so as to indicate the research direction for the significant earthquake event anomaly in the grid area and the period before the large earthquake.

\subsection{Variation characteristics of strain field time factor}

Table 2 Parameters of the strain field temporal factors of ten $M s \geq 7.0$ earthquakes in mainland China

Table 2

\subsection{Relationship between time and space anomalies in strain field}

To analyze the reason of the time-factor anomaly of the strain field ten $M \mathrm{~s} \geq 7.0$ earthquakes in mainland China may be studied from the study area of each earthquake. Take the following as an example, Wenchuan Ms 8.0 earthquake on May 12, 2008, to study the relationship between abnormal time and space. The study area of Wenchuan $M \mathrm{~s} 8.0$ earthquake is $\left(101.5^{\circ}-106.0^{\circ} \mathrm{E}, 30.0^{\circ}-33.5^{\circ} \mathrm{N}\right)$, and the time period for selecting seismic data is from January 1, 1995 to May 11, 2008. The study area is divided into square grids of $0.5^{\circ} \times 0.5^{\circ}$, and 63 grids are divided, and these grids are named. The naming of this paper is defined from bottom to top (latitude division) and left to right (longitude division). Name the grid number (left in Fig. 2). The natural energy orthogonal factor method is used to calculate the seismic energy time factor curve of 63 grids. The results show that the seismic energy factor timing anomalies in the three grids 49, 5, and 1 are abnormal (Fig.3), and Fig.1 (g) The anomalous morphology and anomaly time of the four strain field time factors before Wenchuan $M \mathrm{~s} \mathrm{8.0}$ earthquake are basically the same, while the anomalies of the energy time series curves in other grids 
are not obvious or abnormal. The three grids $\boldsymbol{E}_{i 49} 、 \boldsymbol{E}_{i 5}$ and $E_{i 1}$ are reconstituted into an energy matrix as an anomaly matrix.

$$
E=\left[\begin{array}{rrr}
E_{1,49} & E_{1,5} & E_{1,1} \\
E_{2,49} & E_{2,5} & E_{2,1} \\
\mathrm{M} & \mathrm{M} & \mathrm{M} \\
E_{m, 49} & E_{m, 5} & E_{m, 1}
\end{array}\right]
$$

According to the previous abnormal case study of the strain field space before the large earthquake, the contour value of the strain field in the study area before Wenchuan Ms 8.0 earthquake is calculated (Fig.3), and the isoline value (or absolute value) is also greater than $0.05 \times 10^{5}$. The area of $10^{5}$ value is defined as the anomalous area(Yang, 2017), and the resulting strain field contour abnormal position corresponds to the 49th, 5th, and 1st grid of the study area grid unit. It is indicated that the anomalies of these three grid elements may be the direct cause of the anomalous change of the time factor of the four strain fields before Wenchuan $M \mathrm{~s} 8.0$ earthquake, which further indicates the reliability of the abnormal location of the strain field before Wenchuan $M$ s 8.0 earthquake.

Fig.3

Fig.3 The Wenchuan $M$ s8.0 earthquake study area meshing and naming (left), strain field space contour (right)

Fig.4

Fig.4 Seismic energy timing curves in the 49th, 5th, and 1st grids

\subsection{Space characteristics of strain field}

Fig.5

Fig.5 Distribution of anomalous meshes before the seismic strain field of the $M s \geq 7.0$ earthquakes

According to the response of the strain field time anomaly to the anomaly in the study area grid, the strain field space anomalies of the ten $M s \geq 7.0$ earthquakes in the Chinese mainland are given respectively (Fig. 5). The small circles in Fig.5 represent grid space anomalies. The abnormal position has the following relationship with the main shock epicenter. Before most of the $M \mathrm{~s} \geq 7.0$ earthquakes main shocks, strain field anomalies occurred in the grid around the epicenter, such as the $M$ s 7.1 earthquake in Wuqia, 1985, the Ms 7.6 earthquake in Lancang-Gengma, 1988, and a few earthquake cases. There was no abnormality in the surrounding grid before the earthquake, but there were abnormalities in the grid around the main shock epicenter, such as the $M$ s 7.0 earthquake in Gonghe, 1990, and the Ms 8.0 earthquake in Wenchuan, 2008.

\subsection{Relationship between strain field anomalies and seismic activity}

By analyzing the location of the grid anomaly (small circle), it is possible to explore the seismic activity anomalies in the large earthquake research areas. Taking Lushan Ms 7.0 earthquake on April 20, 2013 as an example, compare the strain field grid anomaly in Fig 5(i) and the time factor anomaly in Fig 2(i). It is found that the No. 2 grid anomaly is caused by the $M_{\mathrm{L}} 4.6$ earthquake group in Quantian County on October 19, 2012. This earthquake group event can be regarded as the short-term anomalous activity before Lushan $M$ s 7.0 earthquake, On February 19, 2013, an $M_{\mathrm{L}} 5.3$ earthquake occurred near Santai County, in Sichuan Province. The earthquake was about 2 months away from Lushan Ms 7.0 earthquake. It can be regarded as a direct foreshock activity of the Lushan earthquake, with short-term anomaly significance, until Lushan about one month before the $M$ s 7.0 earthquake, Wenchuan $M_{\mathrm{L}} 4.1$ 
earthquake occurred on March 4, 2013 in the No. 7 grid. The No. 12 grid occurred on April 8, 2013, Chongqing $M_{\mathrm{L}} 4.3$ earthquake. There are some controversies in the understanding of the 2 earthquakes. Some people think that it is the aftershock of Wenchuan Ms 8.0 earthquake in 2008. Some people think that it is the foreshock of Lushan Ms 7.0 earthquake (Table 3), while other tables are not significant before Lushan Ms 7.0 earthquake in 2013. Moderate earthquake activity.

Table 3 is the correspondence table between the strain space anomalies before the $M \mathrm{~s} \geq 7.0$ earthquakes in China and the seismic activity anomalies. The table shows the grid in which the anomaly is located, the significant earthquakes and swarm events that occur within the grid.

Table 3 Distribution of seismic activity anomalies in the study area before $M s \geq 7.0$ earthquakes strain field

Table 3

It can be seen from Table 3 that the seismic activity of each study area before the $M s \geq 7.0$ earthquake in mainland China is abnormal. The anomalies of a few earthquake cases are several hundred kilometers away from the epicenter of the main earthquake, and there is no abnormality near the extreme earthquake zone. For example, Wenchuan Ms 8.0 earthquake in 2008 and Gonghe Ms 7.0 earthquake in 1990. The seismic activity of most earthquake cases is concentrated around the epicenter, such as Lancang-Gengma Ms 7.6 earthquake in 1988 and 1996 Lijiang Ms 7.0 earthquake in 1966.

\section{Discussion and conclusion}

\subsection{Discussion on whether to remove aftershocks}

It has been a controversial topic to calculate the seismic data selected before the seismic strain field to remove the aftershock. In this work, the purpose of studying the space-time anomaly of the strain field before the large earthquake is to choose whether to remove the aftershock may have a certain impact on the results. Taking the 2008 Wenchuan Ms 8.0 earthquake and the 2013 Lushan 7.0 earthquake as an example, the left column (4 time factors) in Fig 6(a) is the time factor of the aftershocks of the $M \mathrm{~s} \geq 5$ earthquake before Wenchuan $M \mathrm{~s} 8.0$ earthquake, Fig 6(a) the right column (4 time factors) is the time factor of the undeleted aftershock. The abnormalities of the time factors are basically the same. The first two time factor anomalies are slightly different, and the latter two time factors are completely identical. Therefore, Wenchuan $M \mathrm{~s} 8.0$ earthquake Aftershocks and non-deleted aftershocks with the $M \mathrm{~s} \geq 5$ earthquakes have little effect on the research results. The left column of Fig 6(b) (4 time factors) is the time factor of the aftershocks of the $M \mathrm{~s} \geq 5$ earthquakes before the $M \mathrm{~s} 7.0$ earthquake in Lushan. The right column (4 time factors) in the right column of Fig. 6(b) is the time factor of the undestroyed aftershocks. It can be seen that the time factor $T_{\text {third }}$ of the aftershocks of the 5th-order earthquake is the same as the $\mathrm{T}_{\text {fourth }}$ anomaly of the unremoved aftershocks, and the abnormalities of the other 3 time factors are completely different. The reason for the analysis is that the study areas of the two major earthquakes overlap. The aftershocks of the 2008 Wenchuan Ms 8.0 earthquake in the overlap zone affected the time factor anomalies before the 2013 Lushan Ms 7.0 earthquake. The same reason, Wenchuan $M$ s 8.0 earthquake The aftershocks affected the time factor anomalies of the Jiuzhaigou Ms 7.0 earthquake in 2017. Therefore, there are overlapping earthquake cases in the study area. It is recommended to remove the aftershocks of the $M \mathrm{~s} \geq 7.0$ earthquakes. Separate earthquake cases in the study area, it is best not to delete aftershocks.

Fig.6

Fig.6 Time-factor curve of strain field for removing aftershocks and unremoved aftershocks before the Wenchuan 
Ms 8.0 earthquake and Lushan Ms 7.0 earthquakes.

\subsection{Comparison of strain field method and energy field method}

Table 4 shows the four typical fields before the 2008 Wenchuan Ms 8.0 earthquake, the 1988 Lancang-Gengma, Yunnan Ms 7.6 earthquake, and the 1990 Gonghe, Qinghai Ms 7.0 earthquake. By comparing, it can be found that the accuracy of the four typical fields before the strain field is significantly lower than that of the energy field.

Table 4 Comparison of fitting accuracy between energy field and strain field

Table 4

Taking the Yunnan Lancang-Gengma Ms 7.6 earthquake on November 6, 1988 as an example, the four typical field time factor anomalies in the regional energy field of the $M$ s 7.6 earthquake were analyzed. These four typical fields have obvious anomalies. It shows that the anomalous typical field has accounted for 0.9865 of the total field. With the strain field unfolding, the first four typical fields have a fitting accuracy of only 0.6800 (Table 4), and the first four strain fields also have time factor anomalies. The first two strain fields also exhibit short-term anomalies before the earthquake. The 5th to 8th typical field time factor curves of the energy field and the strain field are analyzed separately. It is found that the 5th to 8th typical fields of the energy field account for 0.0895 of the total energy field, and the Lancang-Gengma $M$ s 7.6 earthquake also has obvious time anomalies(Fig.7). While the 5-8 typical fields of the strain field accounted for 0.1998 of the total strain field, only the 8th typical field time factor showed a change of 1.9340 from August to October 1985. Other typical fields There were basically no abnormal changes before the earthquake (Fig.8), and there were many earthquake cases with the same results.

\section{Fig.7}

Fig.7 Time-factor curve of the first to fourth typical energy fields of the Yunnan Lancang-Gengma Ms7.6 earthquake in 1988.

\section{Fig.8}

Fig.8 Time-factor curve of the 5th to 8th typical strain field of the Yunnan Lancang-Gengma Ms7.6 earthquake in 1988.

By extracting time factor anomalies from a large number of earthquake cases and comparing the applied strain field and energy field analysis methods, it is found that the strain field has many unique morning advantages. Firstly, the strain analysis is applied. The same seismic and source parameters have much smaller strain values than the energy values. In the orthogonal transformation, the strain field converges slowly and the anomalies are concentrated in the typical field. The study found that the applied strain field analysis, the anomalous changes are mainly concentrated in the first four typical strain fields, and the energy field anomalies may be distributed in more fields. Secondly, the time-factor curve value of the strain field is obviously smaller than the value of the energy field curve, and it is easy to find abnormal changes in seismic activity in a certain period of time. Finally, the anomaly extracted by the strain field is more reliable and comprehensive, and it is easy to find out the seismic activity caused by the anomaly and some other disturbance changes that further exclude the strain field.

\subsection{Conclusion}

In this paper, the natural orthogonal expansion method is applied to study and discuss the 
spatio-temporal anomaly characteristics of the strain field in each study area before the ten $M \mathrm{~s} \geqslant 7.0$ earthquakes in mainland China since 1980, and the conclusions are as follows:

(1) The time factor of the seismic strain field has an abnormal change pattern of sudden rise or sudden drop on the stationary background before the large earthquake.

(2) At least three strain fields in the first four strain fields before the large earthquakes have abnormal changes, and most of them appear in the first four strain fields. The characteristics of this concentrated and multi-component distribution indicate that the more abnormal components have, the more the abnormal reliability is, and the first strain field anomaly accounts for a large proportion of the total field, and the individual strain field anomalies are independent information.

(3) The earliest time of occurrence of earthquake strain anomaly occurred about 3 years before the main shock. Most of the anomalies occur in the first year before the main shock, with significant shortand medium-term anomaly characteristics, while a few appeared before the main shock from January to June. It has obvious short-term anomaly characteristics.

(4) By comparing the similarity between the strain field time factor anomaly and the abnormal shape of the seismic energy time series curve in each grid unit divided by $0.5^{\circ} \times 0.5^{\circ}$ in the study area, the consistency time of the abnormal time is used to find the seismic strain field. The spatial anomaly position of most large earthquakes is distributed around the epicenter of the main shock, within a distance of $90 \mathrm{~km}$; the spatial anomalies of a few large earthquakes are far from the main epicenter, in the range of $150-250 \mathrm{~km}$.

(5) Analysis of the causes of spatiotemporal anomalies in the strain field before the large earthquake may be caused by abnormal seismic activity in the unit grid in the study area, and significant elemental grids with significant earthquakes or earthquakes occur. These seismic activities may be large in the future. The foreshocks of the earthquake, so how to identify the foreshock activity of the strain field anomaly is the focus of further research in the future.

(6) Given the study areas of the $M \mathrm{~s} \geq 7.0$ earthquakes are independent and do not overlap each other, it is better not to remove the aftershocks, such as the Wenchuan $M \mathrm{~s} 8.0$ earthquake. If the seismic data of such large earthquake strain field anomalies are used, meanwhile the study areas overlap each other and a level 7 The aftershocks of the above earthquakes affect another research area of the $M \mathrm{~s} \geqslant$ 7.0 earthquakes, it is better to remove aftershocks of the $M \mathrm{~s} \geq 5.0$ earthquakes or aftershocks the $M \mathrm{~s} \geqslant$ 7.0 ear in overlap, such as the 2013 Lushan Ms 7.0 earthquake and the 2017 Jiuzhaigou Ms 7.0 earthquake. They are caused by the aftershocks of the 2008 Wenchuan $M \mathrm{~s} 8.0$ earthquake. In short, the seismic data about 10 years before huge earthquake should be kept as good as possible and as real as possible.

\section{Declarations}

\section{Funding}

This project was funded by the Spark Program of Earthquake Science and Technology (Grant number XH18052).

\section{Conflicts of interests}

The authors have no relevant financial or non-financial interests to disclose.

\section{Availability of data and material}

The data and materials used in this study can be obtained from the corresponding author upon request. 


\section{Code availability}

Not applicable

\section{Reference}

Cyrillic, K. Z., \& Kai, E. Q.,(1971).Random function theory and its application in hydrology and meteorology( in Chinese). Bejing: China Press Science .

Luo, G. F., \& YANG, M. Z., (2005). Space-time distributed characteristics on energy field of earthquake in the Yunnan Region. Earthquake research in china, 21(3): 13-18.

Luo, G. F., Yang, M. Z., Ma, H. Q.,et. al. 2011.Intermediate and short-term anomalies of seismic activity energy field before the Wenchuan 8.0 earthquake. Earthquake (in Chinese), 31(3):135-142.

Luo, G. F., Liu, Z. W., Ding, F. H., Ma, H. Q., \& Yang, M. Z. (2018). Research on the seismic strain field prior to the 2017 Jiuzhaigou, Sichuan Ms7.0 earthquake. China Earthquake Engineering Journal, 40(6), 1322-1330.

Luo, G. F., Liu, Z. W., Luo, H. Z.\& Ding F. H. (2019b). Effect of the strain field of Wenchuan Ms 8.0 earthquake on the strong earthquake around the epicenter. Progress in Geophysics (in Chinese), 34(3), 908-918.

Ma, H. Q., \& Yang, M. Z.,(2012).Research on the energy field about Yushu Ms 7.1 earthquake in Qinghai in 2010. Journal of seismological research (in Chinese), 35(4):487-490.

Ma, K. Q., Ding, Y. G., \& Tu, Q.(1993). Principles and methods of climate statistics (in Chinese). Bejing: China Meteorological Press.

Yang, M. Z., \& Zhao, W. M., (2004). Statistical analysis on energy field of seismicity in Ningxia and its neighborhood region. Acta Seismologica Sinica (in Chinese), 26(5): 516-522.

Yang, M. Z., \& Ma, H. Q., (2011).Variation of energy field of Longmenshan fault zone before the Wenchuan Ms8.0 earthquake. Earthquake research in china,27(3):260-267.

Yang, M. Z., \& Ma, H. Q., (2012). Analysis of regional seismic energy field before Wenchuan Ms8.0 earthquake. Progress in Geophysics (in Chinese), 27(3):0872-0877.

Yang, M. Z., \& Ma, H. Q., (2013). Features of Time Factor Anomalies of regional energy field before large earthquake. Earthquake (in Chinese), 33(3):107-115.

Yang, M. Z., Ma, H. Q., Luo, G. F., et. al.(2017). Research on the seismic strain field before strong earthquakes above $M \mathrm{~s} \geq 6$ in Chinese mainland. Geophysics (in Chinese).60(10):3804-3814. 


\section{Figures}

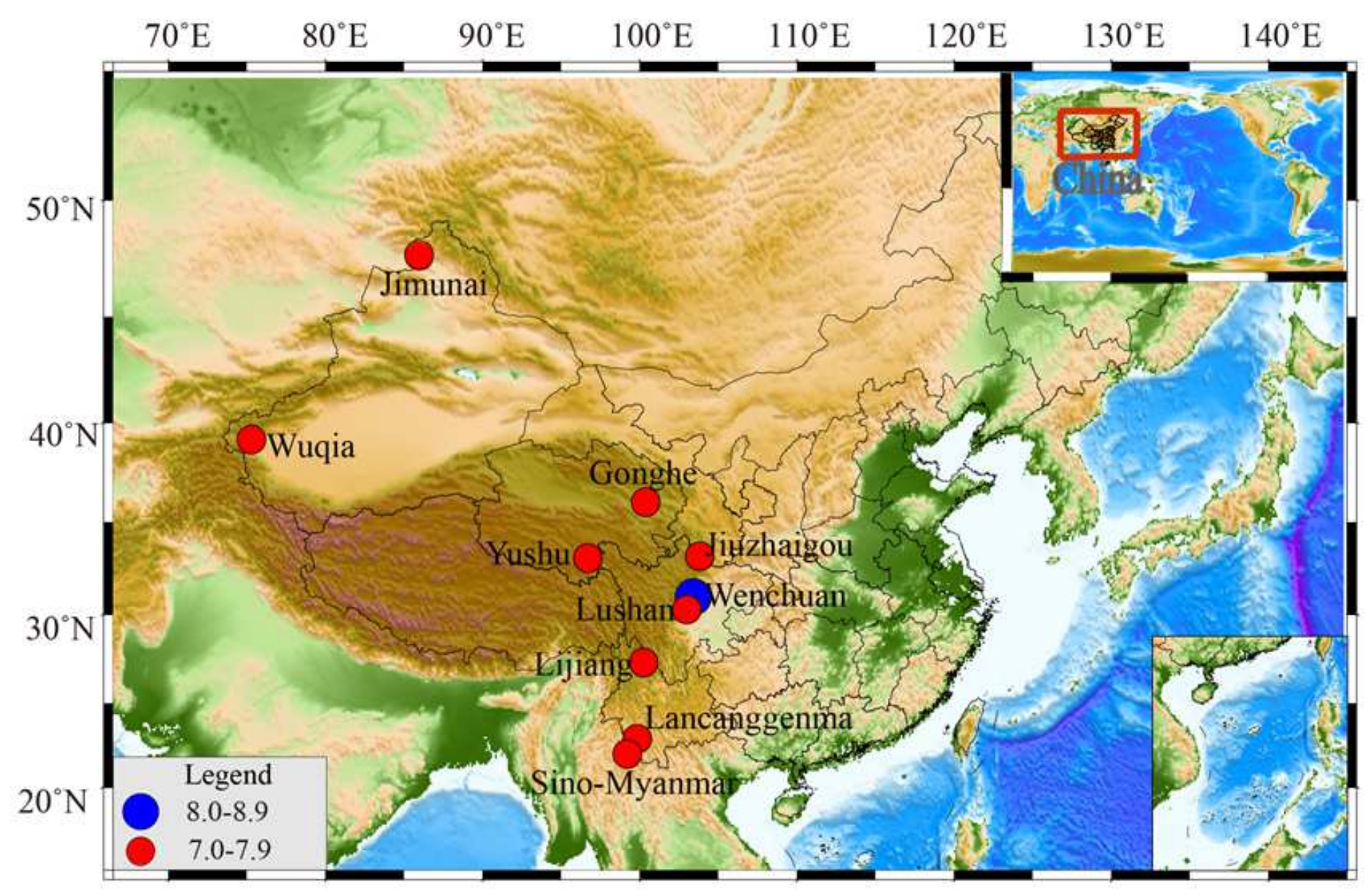

\section{Figure 1}

Study areas of ten Ms $\geq 7.0$ earthquakes in mainland China Note: The designations employed and the presentation of the material on this map do not imply the expression of any opinion whatsoever on the part of Research Square concerning the legal status of any country, territory, city or area or of its authorities, or concerning the delimitation of its frontiers or boundaries. This map has been provided by the authors. 

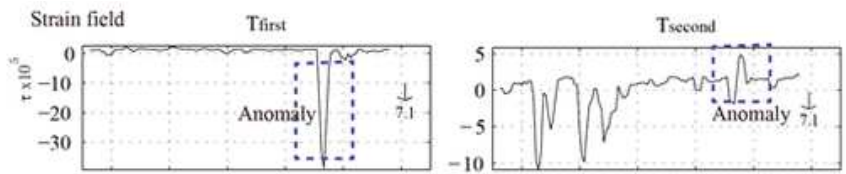

$\begin{array}{llllllllllll}1976 & 1978 & 1980 & 1982 & 1984 & 1986 \text { year } & 1976 & 1978 & 1980 & 1982 & 1984 & 1986 \text { year }\end{array}$
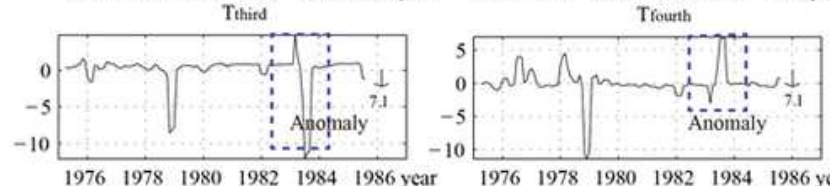

(a) Wuqia Ms7.1 earthquake in 1985
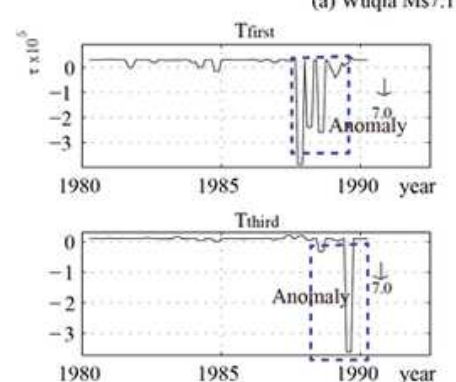

(c) Gonghe Ms7,0 earthquake in 1990
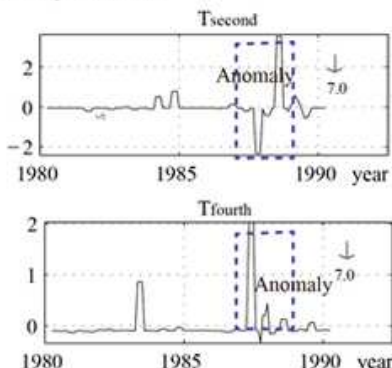

1990 year

Tsecond

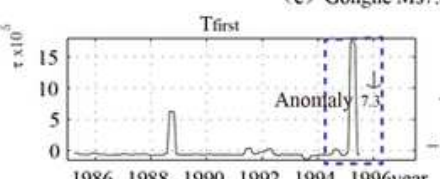

$19861988 \quad 1990 \quad 1992 \quad 1994 \quad 1996 y c a r$
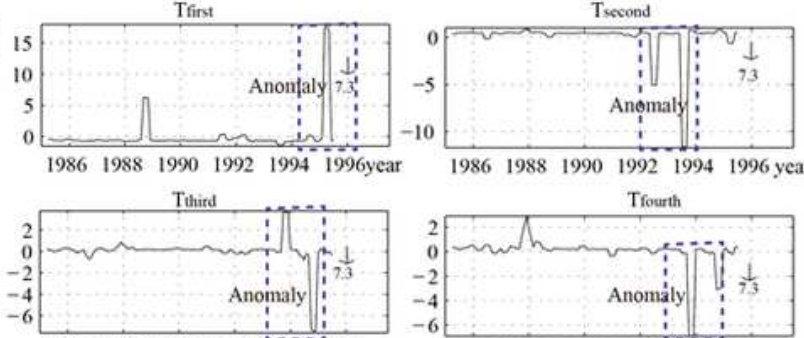

$\begin{array}{lllll}1986 \quad 1988 & 1990 \quad 1992 \quad 1994 \quad 1996 \text { year }\end{array}$

Tfourth

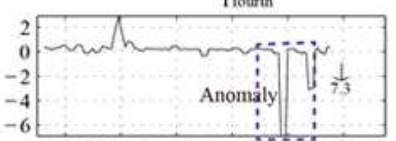

$\begin{array}{llllllllllll}1986 & 1988 & 1990 & 1992 & 19994 & 1996 y e a r & 1986 & 1988 & 1990 & 1992 & 1994 & 1996 \text { yea }\end{array}$ $T_{\text {first }}$ (e) China borders Ms7.3 carthquake in 1995 Tsecond
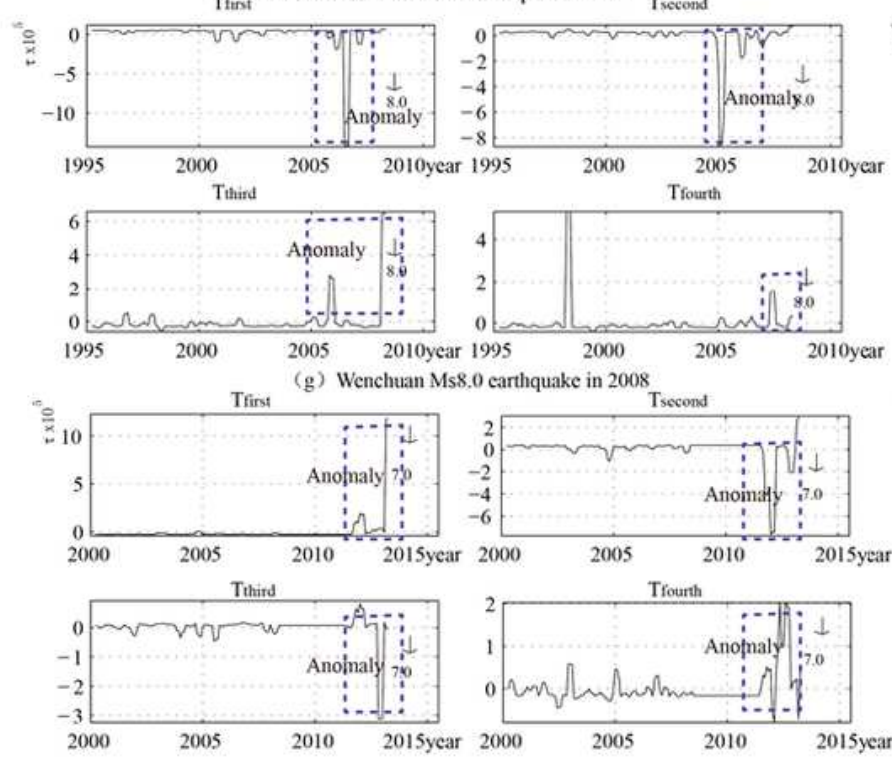

(i) Lushan Ms7.0 earthquake in 2013
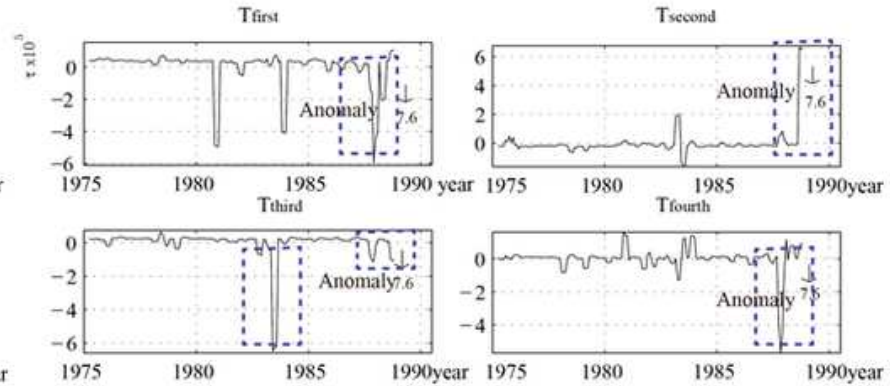

(b) Lancang Ms7.6 earthquake in 1988
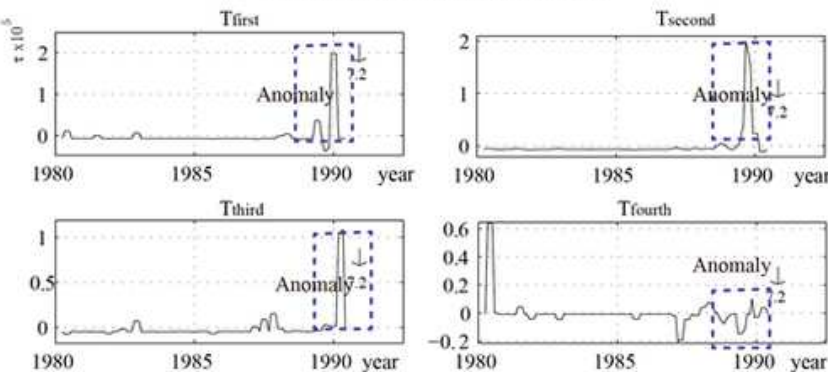

(d) Jimuna Ms7.2 earthquake in 1990
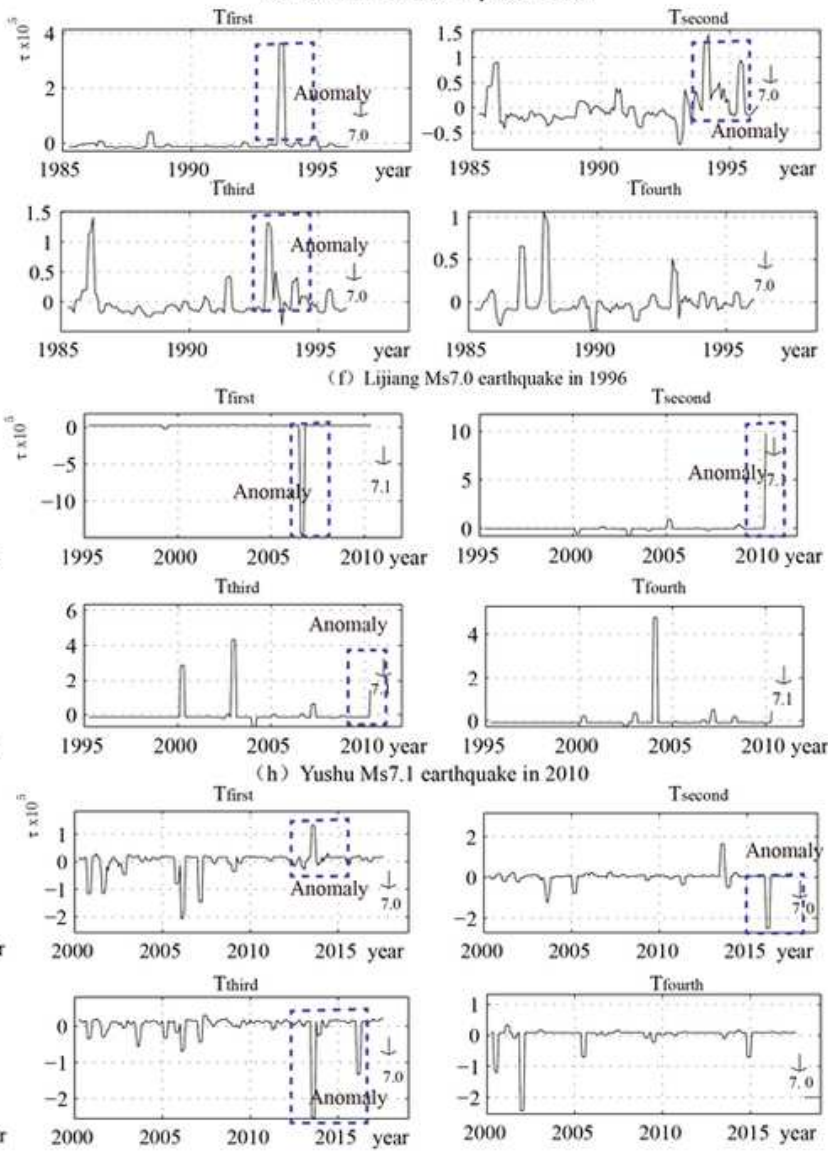

(j) Jiuzaigou Ms7.0 earthquake in 2017

\section{Figure 2}



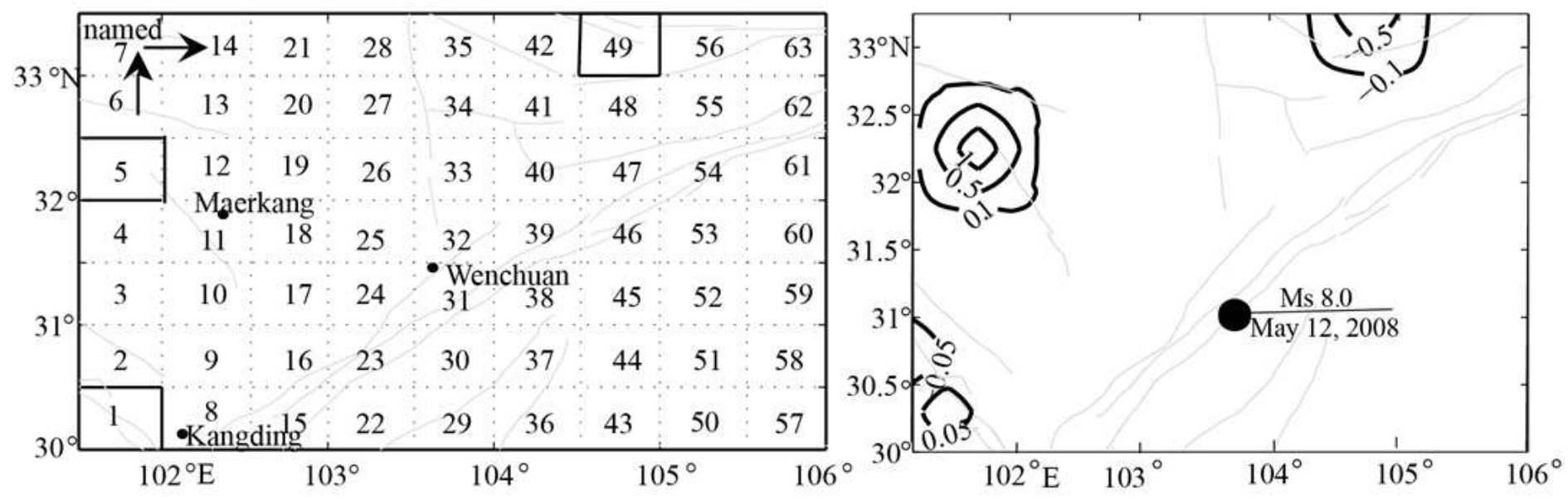

- County nane $\rightarrow$ named the direction $\quad .05$ Isolone Contour times 10 to the fifth power

Figure 3

The Wenchuan Ms8.0 earthquake study area meshing and naming (left), strain field space contour (right)
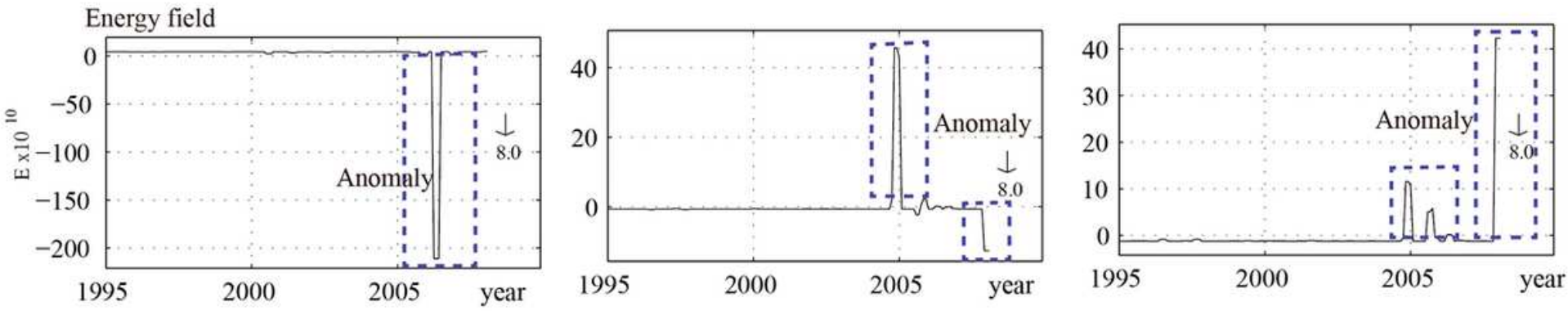

Figure 4

Seismic energy timing curves in the 49th, 5 th, and 1 st grids
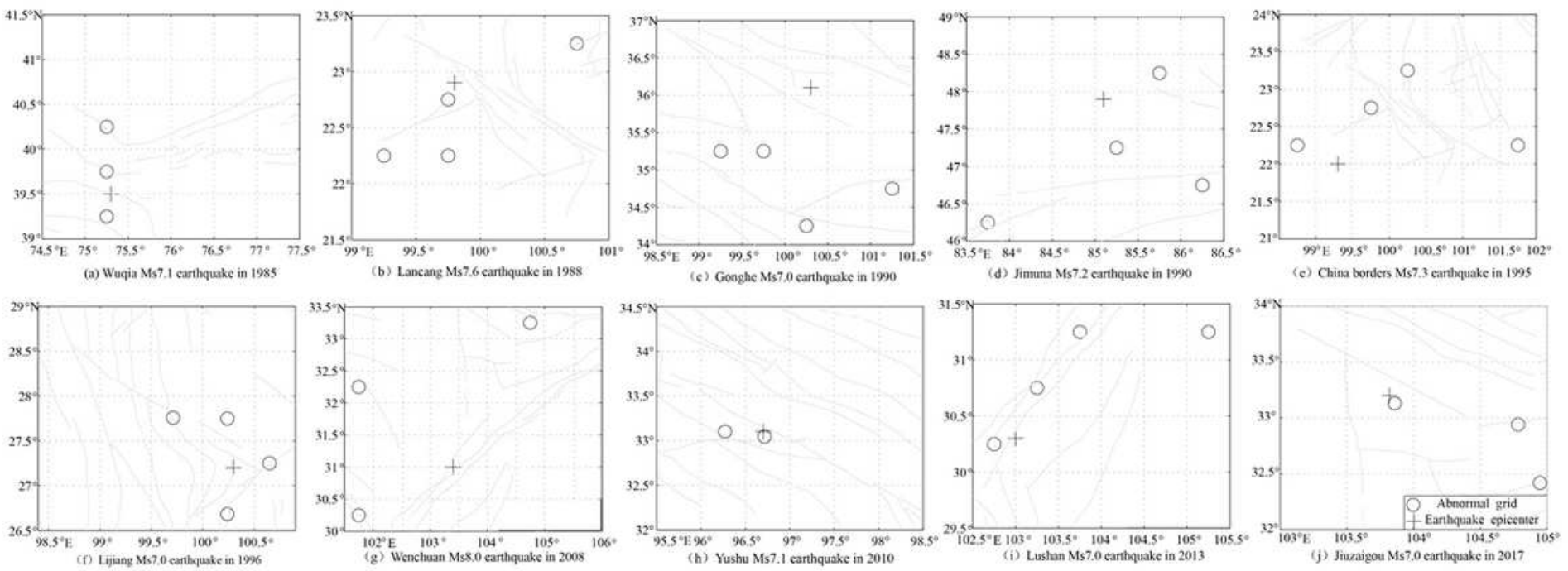

Figure 5 
Distribution of anomalous meshes before the seismic strain field of the Ms $\geq 7.0$ earthquakes

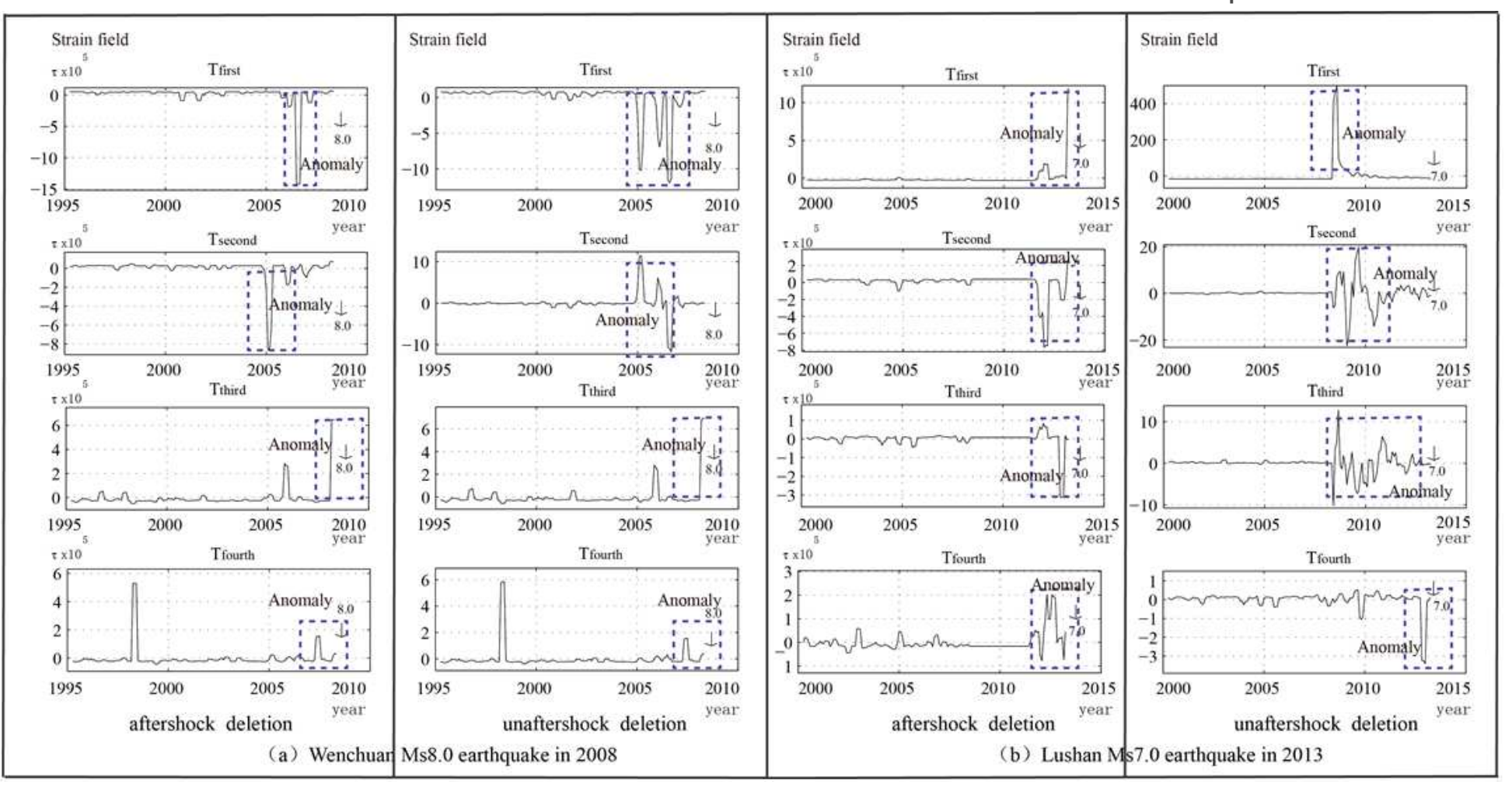

\section{Figure 6}

Time-factor curve of strain field for removing aftershocks and unremoved aftershocks before the Wenchuan Ms 8.0 earthquake and Lushan Ms 7.0 earthquakes.

Energy field

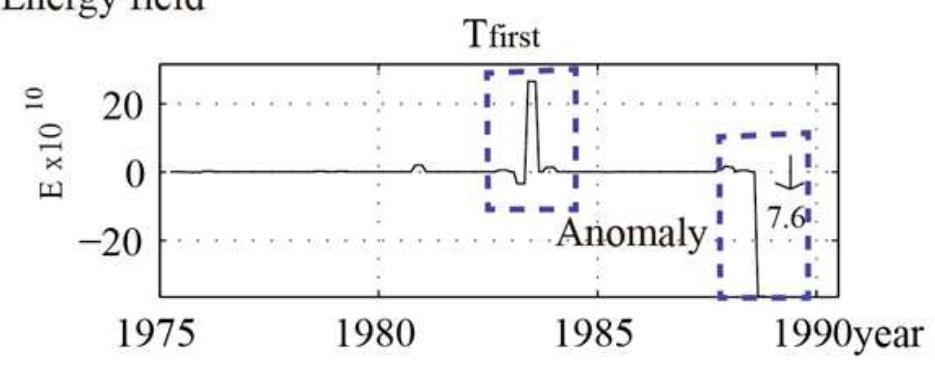

Tthird

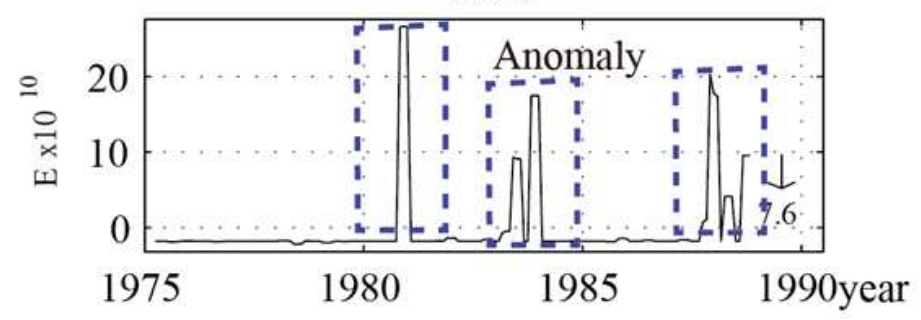

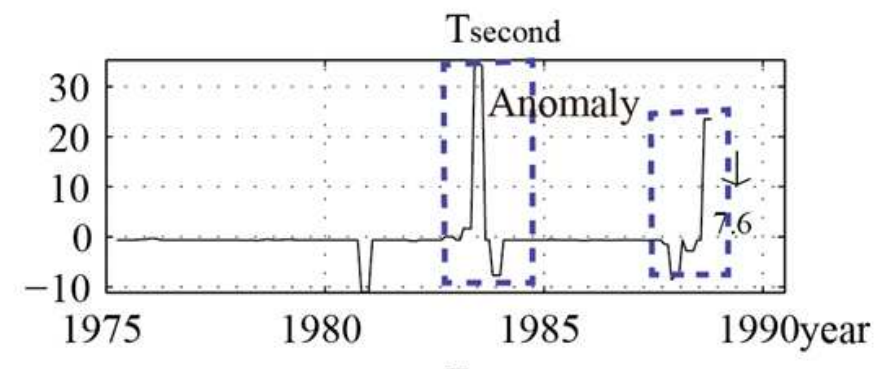

Tfourth

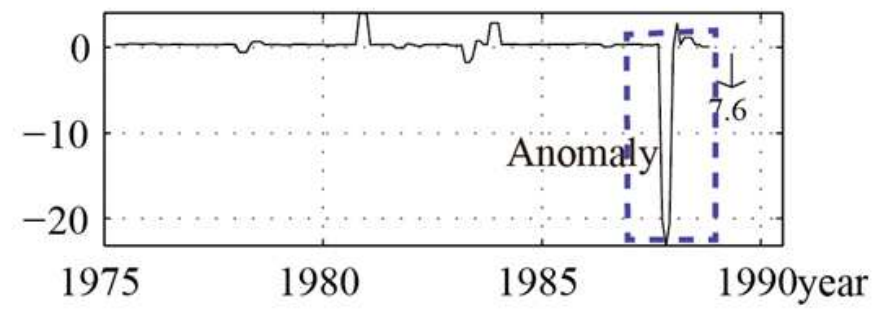

Figure 7

Time-factor curve of the first to fourth typical energy fields of the Yunnan Lancang-Gengma Ms7.6 earthquake in 1988. 


\section{Strain field}
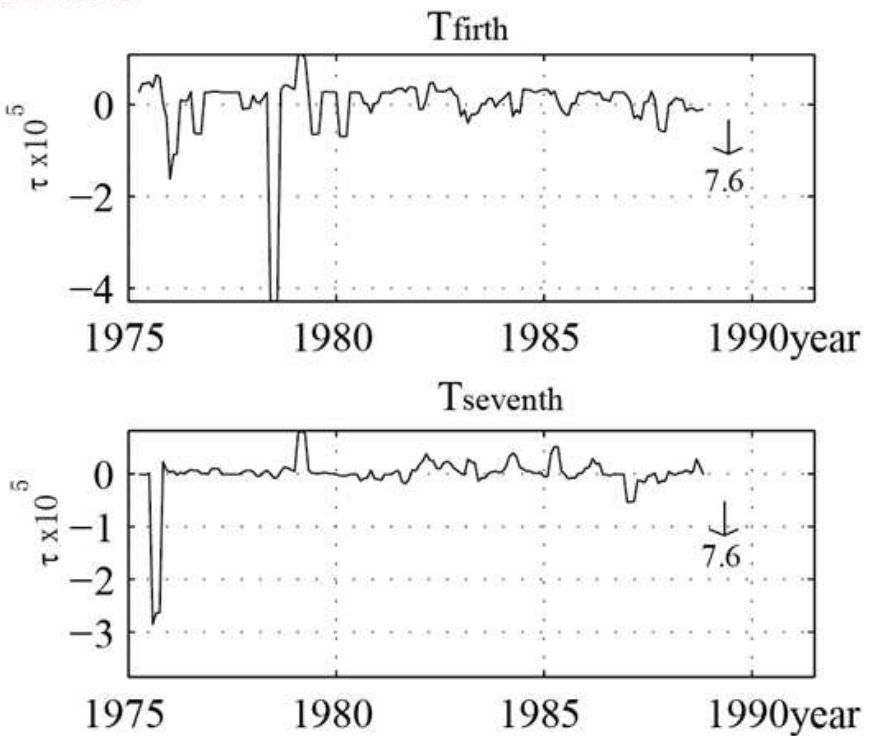

Tsixth
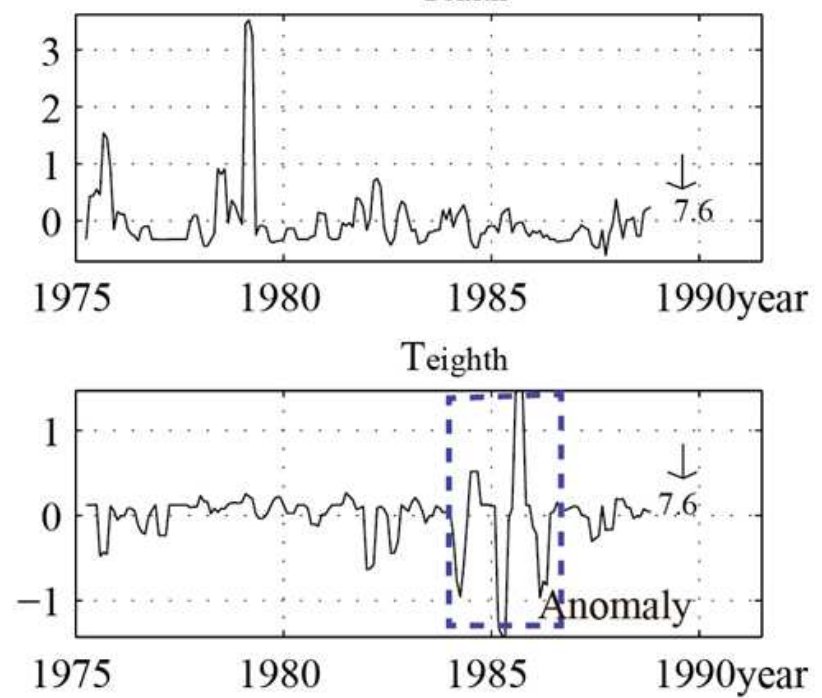

\section{Figure 8}

Time-factor curve of the 5th to 8th typical strain field of the Yunnan Lancang-Gengma Ms7.6 earthquake in 1988. 